\title{
Absence of overshadowing in rats with hippocampal lesions
}

\author{
NESTOR A. SCHMAJUK, NORMAN E. SPEAR, and ROBERT L. ISAACSON \\ State University of New York, Binghamton, New York
}

\begin{abstract}
Rats with hippocampal, cortical, and sham lesions were trained in two simultaneous discriminations: one between two odors, and the other between two visual stimuli. One odor signaled shock and another odor "no shock," both with probability of 1 . A visual stimulus signaled shock with probability of 1 , but the other visual stimulus signaled "no shock" only twothirds of the time. In this situation, the odor dimension overshadowed the visual dimension in sham animals but not in the hippocampal-lesioned animals. No assessment can be made of the behavior of animals with only neocortical damage, since they did not learn the visual stimulus-shock association even when the visual stimulus was given alone. These results support a description of some of the sequelae of the hippocampal lesions in terms of attentional deficits.
\end{abstract}

Some of the sequelae of hippocampal lesions can be explained in terms of attentional changes. For example, after hippocampectomy, latent inhibition is disrupted (McFarland, Kostas, \& Drew, 1978; Solomon \& Moore, 1975), blocking is attenuated (Rickert, Bennett, \& French, 1978; Solomon, 1977), and overshadowing is absent (Rickert, Lorden, Dawson, Smyly, \& Callaham, 1979).

Solomon (1980) explained these failures in terms of the hippocampal involvement in "attentional processes which enable the organism to learn to ignore irrelevant stimuli'" (p. 254). Moore and Stickney $(1980,1982)$ proposed that the hippocampus is essential for the animal's computation of stimulus salience (associative rate) as defined in Mackintosh's (1975) attentional theory.

The present paper attempts to study hippocampallesioned animals in an overshadowing paradigm, using odors and shapes as stimuli. Animals were trained in two simultaneous discriminations. One discrimination was between two odors and the other was between two visual stimuli. This procedure differed from that of Rickert, Lorden, et al. (1979), who used two simultaneous stimuli. In our experiment, one odor, $\mathrm{S}+$, signaled shock and another odor, S-, signaled "no shock," both with probability of 1 . A visual stimulus, $\mathrm{S}+$, signaled shock with probability of 1 , but the other visual stimulus, S-, signaled "no shock" only two-thirds of the time. On this basis, the odor stimuli were more reliable predictors of shock than were the visual stimuli (see Table 1).

These contingencies were obtained by distributing

The first author is a Fellow of the Consejo Nacional de Investigaciónes Cientificas y Tecnicas de la Republica Argentina. The authors' mailing address is: Center for Neurobehavioral Sciences, Department of Psychology, State University of New York at Binghamton, Binghamton, New York 13901. the odor and visual stimuli over 20 training trials in such a way that a lemon odor (odor $\mathrm{S}+$ ) and vertical stripes (visual $\mathrm{S}+$ ) were presented together five times, and on every trial they preceded footshock. On five other occasions, the lemon odor (odor $\mathrm{S}+$ ) was presented with horizontal stripes (visual $\mathrm{S}-$ ), and this combination also preceded footshock. On the 10 other trials, a methyl odor (odor $\mathrm{S}-$ ) was paired with horizontal stripes (visual $\mathrm{S}-$ ), and this combination was followed by footshock. Thus, the lemon odor and vertical stripes were always associated with footshock, the methyl odor was never associated with footshock, and the horizontal stripes were associated with footshock in an inconsistent fashion, predicting shock one-third of the time.

In this situation, the odor dimension should overshadow the visual dimension because it predicts the presence of shock better than does the visual. In this case, according to Mackintosh's (1975) theory, the "associative rate" for odors (or for methyl alone) should be greater than that for visual input (or for horizontal stripes alone). Since, according to Moore and Stickney (1980), the "associative rate" in hippocampal lesioned animals does not change, no overshadowing should be present.

\section{METHOD}

\section{Subjects}

The subjects were 55 male, 90-day-old (at the beginning of the experiment), Sprague-Dawley-derived albino rats, bred and reared in the animal colony at SUNY-Binghamton. They were individually caged, with water and food available ad lib. A 12 12 light-dark cycle was observed in the colony room, and training and testing were performed from 4 p.m. to 6 p.m.

\section{Surgical Procedures}

Animals were assigned at random to one of the three groups. The hippocampal group received bilateral aspiration of the hippocampus and the overlying cortex (Isaacson \& Woodruff, 1976). 
The cortical group received bilateral aspiration lesions of the same part of the neocortex that had been aspirated in the preceding group. A sham group received only two burr holes in the skull at the same place as had been made in the previous groups when surgery was begun. The rats were allowed 30 days for postoperative recovery.

\section{Apparatus}

Four white, translucent Plexiglas chambers were used in training; each measured $22 \mathrm{~cm}$ long $\times 14 \mathrm{~cm}$ wide $\times 15 \mathrm{~cm}$ high. Two of the chambers had 16 black vertical stripes $2 \mathrm{~cm}$ wide and $2 \mathrm{~cm}$ apart, and the other two had 4 black horizontal stripes of the same size and separation. Each chamber had a grid floor through which a 0.4-mA scrambled footshock, provided by a Grason-Stadler shock generator, could be delivered. Clear Plexiglas trays with lemon- or methyl-salicilate-soaked cotton could be fitted under the grids of each chamber when appropriate. Four odor-visual combinations resulted: (1) methylvertical, (2) methyl-horizontal, (3) lemon-vertical, and (4) lemonhorizontal.

Two testing apparatuses were used. The vertical-horizontal preference was measured in a two-chamber Plexiglas apparatus. One chamber had vertical stripes and one had horizontal stripes of the same dimensions as the training chambers; it also had a grid floor. The lemon-methyl preference was evaluated in a clear Plexiglas cage, $44 \mathrm{~cm}$ long $\times 14 \mathrm{~cm}$ wide $\times 15 \mathrm{~cm}$ high, with one roll (14 cm long, $3 \mathrm{~cm}$ in diameter) on each end covered with fur soaked with the odor essences.

For the application of the noncontingent footshock, a chamber $30 \mathrm{~cm}$ square and $60 \mathrm{~cm}$ high, with a grid floor, was used.

\section{Procedure}

The subjects were assigned randomly to one of nine groups, according to the lesion (SHAM, CORTICAL, or HIPPOCAMPAL), and the treatment (CONTROL, VISUAL, EXPERIMENTAL). The number of subjects for each group was: SHAM-CONTROL (4), VISUAL (5), EXPERIMENTAL (6); CORTICAL-CONTROL (5), VISUAL (7), EXPERIMENTAL (9); HIPPOCAMPAL-CONTROL (7), VISUAL (6), EXPERIMENTAL (6). All groups were handled during the 3 days preceding the experiment.

Training was carried out in the training apparatus. The experimental groups were exposed to one odor stimulus and one visual stimulus on each training trial, according to the odor and visual contingencies of Table 1 . Twenty trials, each 1 min long, were given. In 10 of them, the animals received two shocks of $0.4 \mathrm{~mA}$ and $1-\mathrm{sec}$ duration separated by $20-30 \mathrm{sec}$. The trials on which shock was given were determined randomly.

The visual-only groups were exposed only to the "visual-only" contingencies of Table 1 without odors under the training cages, but received the same number of trials in the same order as the experimental groups. One hour after training, these groups were exposed for $2 \mathrm{~min}$ to each odor in a different room and cage.

The control groups were exposed to the same manipulation of the experimental groups with visual stimuli and odors, but no shock was delivered. One hour after this procedure, 20 noncontingent footshocks of the same intensity and duration as for experimentals were delivered in a different room and cage.

Preference tests were given $24 \mathrm{~h}$ later. Each animal was placed

Table 1

Contingency Tables for Odors and Visual Shapes

\begin{tabular}{crrllr}
\hline Odor & S & NS & Visual Shape & S & NS \\
\hline Lemon & 10 & 0 & Vertical & 5 & 0 \\
Methyl & 0 & 10 & Horizontal & 5 & 10 \\
\hline
\end{tabular}

Note $-S=$ shock; NS = nonshock. Numbers indicate number of times each stimulus was presented with or without shock. in the center of the testing apparatus, and the time spent on each side (vertical vs. horizontal, lemon or methyl half) was recorded for $3 \mathrm{~min}$. Tests were given $30 \mathrm{~min}$ apart. The test for visual preference was always given first, since this was during the most sensitive part of the experiment and we wanted to avoid possible extinction effects due to a prior exposure to the testing cage.

\section{Histological Analyses}

When behavioral analyses were completed, each rat was sacrificed with an overdose of chloroform and was perfused through the left ventricle of the heart with $0.9 \%$ saiine followed by $10 \%$ Formalin. The brains were fixed in Formalin, and frozen sections were cut at $30 \mu$. Every sixth section was retained, mounted, stained with thionine, and examined for the extent of the lesion.

\section{RESULTS}

\section{Histology}

Figure 1 shows drawings of the largest and smallest hippocampal and cortical lesions. In the first case, removal of the hippocampus was almost complete, with no damage of the underlying thalamic nuclei.

\section{Behavior}

The upper panel of Figure 2 shows the mean percentage of time (preference) spent by the nine groups of animals on the side of the vertical stripes, when tested for the amount of time spent on the sides with vertical or horizontal stripes, in the testing cage. The lower panel shows the preference for the lemon odor relative to the methyl. Both panels indicate the animal's preferences for vertical stripes or lemon, after giving the three surgical groups the different types of training: control procedures, visual training only, and both visual and odor training.

In the odor choice situation, the effect of visual and odor training was to reduce the preference for the lemon in all surgical groups $[F(2,46)=18.43$, $p<.001]$, and the effect of training did not change under the different lesion conditions (no interactions, $\mathrm{F}<1$ ). It also can be seen that the lesion modified the average preference for the odors, since without training, or after receiving visual training only, hippocampals seem to have preferred the lemon to a greater extent than did the other groups $[\mathrm{F}(2,46)$ $=4.39, \mathrm{p}<.025]$.

In the visual choice situation, the effect of training on the different lesioned groups was different $[F(4,46)=2.69, p<.05]$. The sham group evidenced overshadowing, since, even though the effect of training was significant when the visual stimuli was presented alone $[F(1,46)=4.69, \mathrm{p}<.05]$, no significant learning was attained when odors and visual patterns were given together $(F<1)$. For the cortical group, the outcome is not clear, since these animals did not learn about the visual stimuli even when presented alone (nonsignificant simple main effect). The hippocampal group did not show over- 


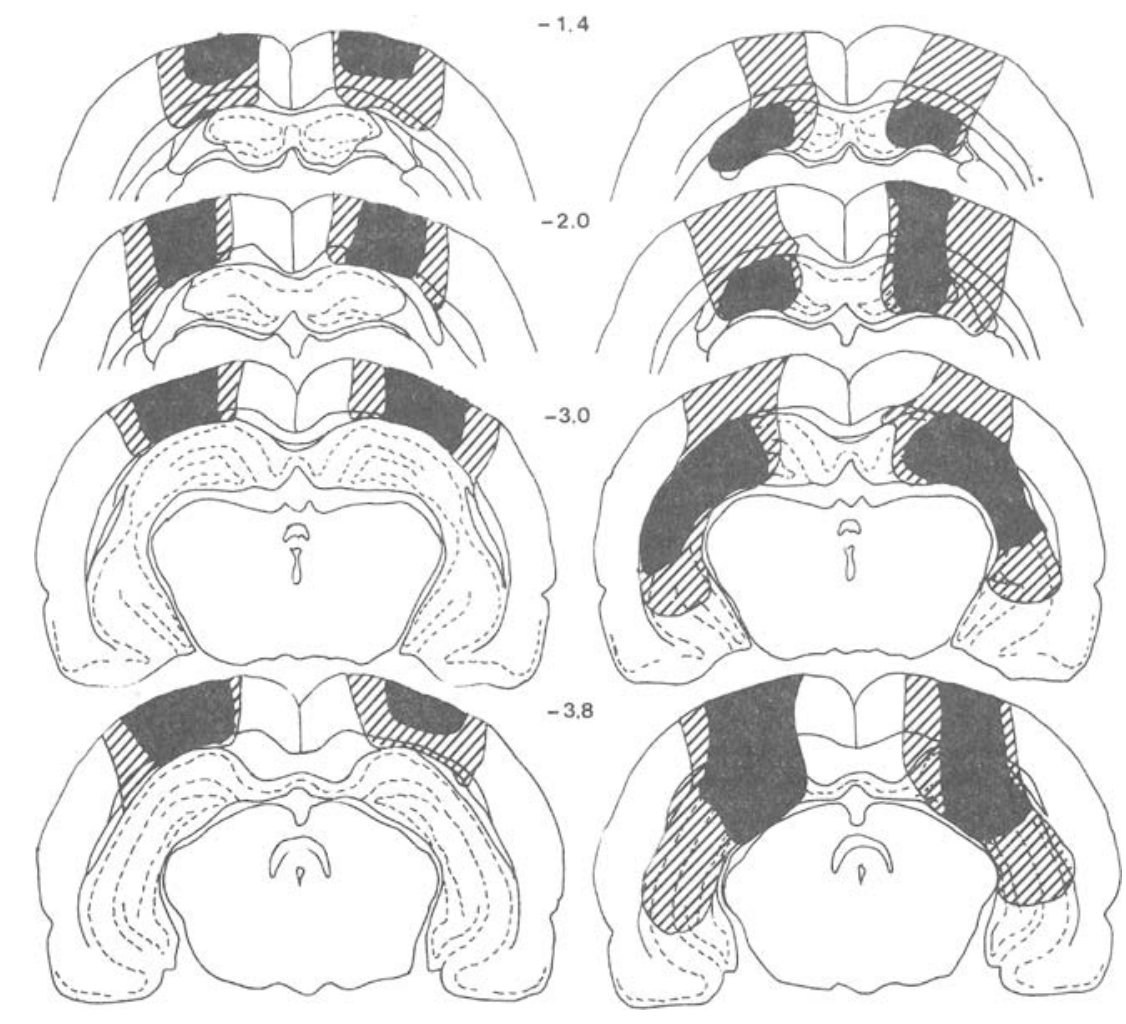

Figure 1. Extent of the lesions in rats with cortical (right) and hippocampal (left) ablations. Lined areas show the maximal and black areas the minimal extents of the lesion.

shadowing, because although they did learn about the visual stimuli when these were presented alone $[F(1,46)=9.87, \mathrm{p}<.01]$, they also showed learning when the visual stimulus was presented with the odor $[F(1,46)=9.12, p<.01]$. The effect of training on odor and visual stimuli produced a significant interaction $[F(1,46)=4.56, p<.01]$ because of the reduction in time on the vertical side found in the hippocampal lesion group.

\section{DISCUSSION}

The effect of hippocampal lesions was assessed in animals trained in two simultaneous discriminations between two odors and two visual stimuli. The animals with hippocampal lesions did not show overshadowing and learned equally well about two sets of stimuli. In the same conditions, sham-lesioned animals showed an overshadowing of visual stimuli by odors.

As was pointed out before, Moore and Stickney (1980) proposed that the hippocampus would change the associability of stimuli, according to Mackintosh's (1975) attention theory, and applied the model to latent inhibition and blocking after hippocampal lesions. Recently, Moore and Stickney (1982) applied the model to explain deficits in spatial learning shown by hippocampal-lesioned animals.
In the overshadowing paradigm, the model predicts that a stimulus having either greater salience (associative rate, $\alpha$ ) or a higher rate of reinforcement will achieve more associative strength and therefore overshadow the other stimulus. In our experiment, because the odors had higher salience, contingency, or both, they overshadowed the visual stimuli in the sham animals. Since the as could not be modified in the hippocampal animals, no overshadowing should be observed according to the Moore and Stickney model.

An alternative explanation, that is, that the hippocampal animals do not show overshadowing simply because they are superior learners for visual stimuli, can be rejected because hippocampal and sham animals given only visual learning did not differ. (F interaction for hippocampal and sham groups, in control and visual training only situations, is less than 1.)

As mentioned, the predictive values for individual stimulus were P US | methyl $=0$ and $P$ US | horizontal $=.33$, and since methyl is the most reliable predictor of nonshock, it should overshadow the preference for horizontal. However, the predictive value of each dimension can be calculated as a root mean square contingency (Gibbon, Berryman, \& Thompson, 1974), resulting for odors $\phi=1$ and for visual stimuli $\phi=.74$, and since the odor dimen- 


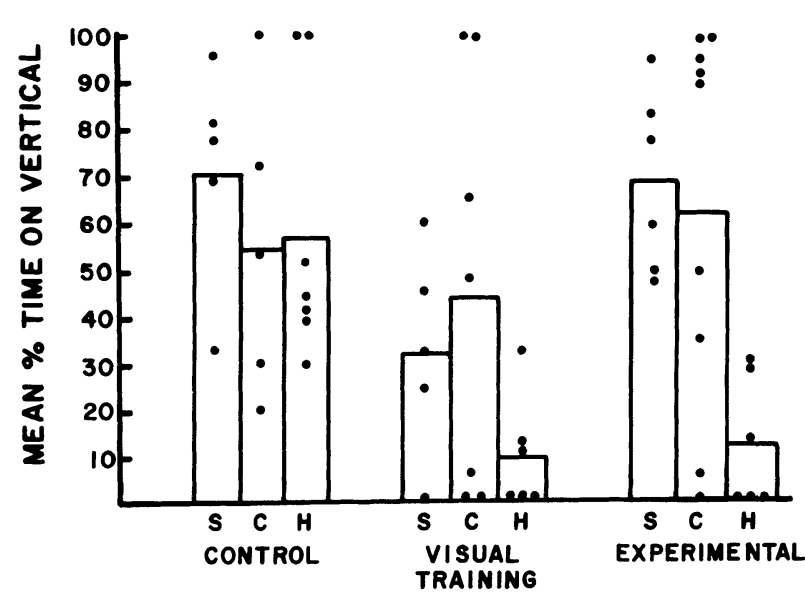

priately to variations of motivational state in a $T$ maze in which food and water can be found in opposite arms (Hirsh, Holt, \& Mosseri, 1978). Only additional assumptions, such as changes in stimulus salience as controlled by the animal's motivational state, could explain these results.

\section{REFERENCES}

Gibbon, J., Berryman, R., \& Thompson, R. L. Contingency spaces and measures in classical and instrumental conditioning. Journal of Experimental Analysis of Behavior, 1974, 21, 585-605.

Hirsh, R., Holt, L., \& Mosseri, A. Hippocampal mossy fibers, motivational states, and contextual retrieval. Experimental Neurology, 1978, 62, 68-79.

IsAACson, R. L., \& WoodruFf, M. L. Spontaneous alternation and passive avoidance behavior in rats after hippocampal lesions. In B. Hart (Ed.), Experimental psychobiology: A laboratory manual. San Francisco: Freeman, 1976.

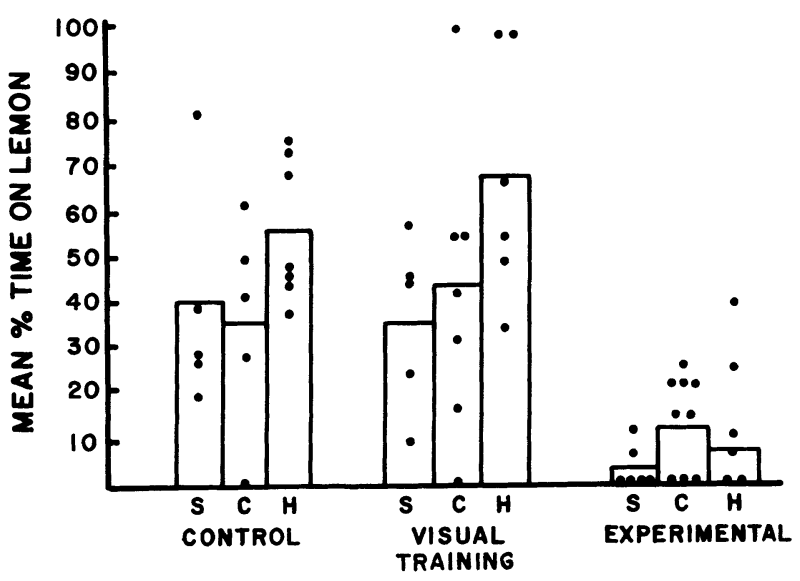

Mackintosh, N. J. A theory of attention: Variations in the associability of stimuli with reinforcement. Psychological Review, 1975, 82, 276-298.

McFarland, D. J., Kostas, J., \& Drew, W. G. Dorsal hippocampal lesions: Effects on preconditioning CS exposure on flavor aversion. Behavioral Biology, 1978, 22, 398-404.

Moore, J. W., \& Stickney, K. J. Formation of attentionalassociative networks in real time: Role of the hippocampus and implications for conditioning. Physiological Psychology, 1980, 8, 207-217.

Moone, J. W., \& Stickney, K. J. Goal tracking in attentionalassociative networks: Spatial learning and the hippocampus. Physiological Psychology, 1982, 10, 202-208.

Rickert, E. J., Bennett, T. L., \& French, J. Hippocampectomy and the attenuation of blocking. Behavioral Biology, $1978,22,597-609$.

Figure 2. Mean percentage time on lemon (bottom panel) or vertical stripes (upper panel) sides in the preference testing boxes. $\mathbf{S}=$ sham-lesioned group; $\mathbf{C}=$ cortical-lesioned group; $\mathbf{H}=$ hippocampal-lesioned group.

sion is more reliable than the visual dimension, the first should overshadow the second. Whether entire dimensions or individual stimuli are playing a role in this case is still a matter of further investigation in the normal animal.

According to these results, explanations based on attentional deficits can predict many of the sequelae of the hippocampal lesions. However, there are other behaviors affected by this lesion that can be described in attentional terms only with difficulty, for example, the failure to respond appro-

Rickert, E. J., Lorden, J. F., Dawson, R., Smyly, E., \& Callaham, M. F. Stimulus processing and stimulus selection in rats with hippocampal lesions. Behavioral and Neural Biology, 1979, 27, 454-465.

Solomon, P. R. Role of the hippocampus in blocking and conditioned inhibition of the rabbit nictitating membrane response. Journal of Comparative and Physiological Psychology, 1977, 91, 407-417.

Solomon, P. R. A time and a place for everything? Temporal processing views of hippocampal function with special reference to attention. Physiological Psychology, 1980, 8, 254-261.

Solomon, P. R., \& Moore, J. W. Latent inhibition and stimulus generalization of the classically conditioned nictitating membrane response in rabbits (Oryctolagus cuniculus) following dorsal hippocampal ablation. Journal of Comparative and Physiological Psychology, 1975, 89, 1192-1203.

(Manuscript received December 19, 1982; revision accepted for publication March 2, 1983.) 